\title{
ACADEMIC CAREER OF \\ 54 CONDENSED MBBS SPECIALISTS
}

\author{
Naima Muazzam
}

\section{Introduction}

In a study of 647 medical specialists of Bangladesh (Muazzam, 1986), there were 54 condensed MBBS doctors who specialised in different branches of medical science. In this paper, the academic career of these condensed doctors is provided.

LMF or Licenciate course was four years after Matriculation in a Medical school. Since during this course proper teaching of all branches of medical Science was not possible, a short course of two years was introduced for the LMF doctors to obtain MBBS degree. These doctors were known as condensed doctors. Now LMF system is abolished.

Results and Discussion : Among the 54 doctors $46(85.19 \%)$ were male and 08 $(14.81 \%)$ were female. Most of them were students of different medical schools in the country and a few were from Indian schools. Maximum member (8) belonged to Dhaka distric followed by 6 each from Mymensingh and Sylhet, 5 from Rajshahi, 4 from Comilla, 3 from Barisal and 2 each from B. Baria, Chittagong, Noakhali and Patuakhali and India.

\section{Age at LMF Gualification}

Table I shows the age distribution of LMF quafications. 10 each were aged 20 and 22 years followed by 8 aged 23 years, 7 aged 19 years and 6 each aged 21 and 24 years. 2 each aged 18 years and 25 years, while I was aged 26 years. Two did not mention their age.

Table 2 shows the age distribution of MBBS quafication of the 54 LMF doctors. The 8 female doctors were aged 26 to 31 years while the male doctors were aged 23 to 47 years. One did not mention the age. Maximum -12 passed at-26 years of age.

The age of obtaining the first post graduate qualification is shown in Table 3.53 of them obtained their first P.G qualification by 30 to 47 years of age while one was aged 53 years. The 8 females were aged 30-45 years. Maximum member 06 was aged 33 years followed by 05 each, aged $30,35,37$ and 42 years, 4 aged 38 years.

Table 4 shows the time gaps between matric/ SSC, and LMF, LMF and MBBS, also MBBS and first post graduate qualifications.

The time gaps could be due to failure of examination or for not continuing study for some other reason. The exact reason was not mentioned.

Table 5 shows the age of marriage of the condensed doctors. A few male doctors married in comparatively younger age-17-22 years, probably for obtaining financial help, otherwise, there is no special significance in the age of marriage. Two of them did not mention the age of marriage.

Table 6 shows the member of children, three doctors had no child. Among the 51 doctors who had children, $43(84,31 \%)$ had 2 to 4 children each. Among the 43 doctors $30(70 \%)$ of them had 3 to 4 children. Only 2 had six children each and one each more had seven and eight children. The total member of children is 182 (vide Table-6). 
Table I. Shows the age- distribution at LMF gualifications

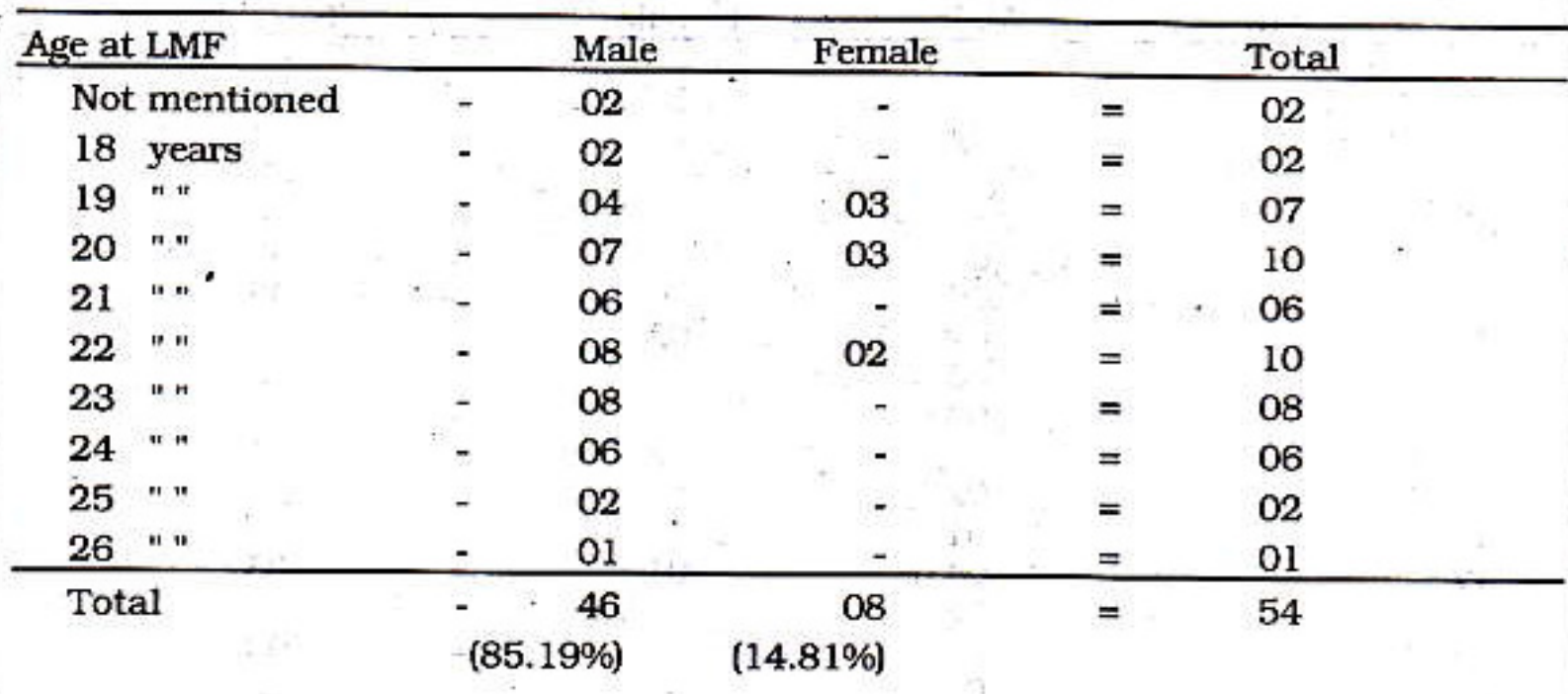

Table 2. showing the age of 54 condensed MBBS doctors

\begin{tabular}{lrrrr}
\hline Age at MBBS & - & Male & Female & Total \\
\hline age not mentoned & - & 01 & - & 01 \\
23 Years & - & 01 & - & 01 \\
$24 "$ & - & 03 & & 03 \\
$25 "$ & - & 02 & - & 02 \\
$26 "$ & - & 10 & 02 & 12 \\
$27 "$ & - & 03 & 02 & 05 \\
$28 "$ & - & - & - & - \\
$29 "$ & - & 03 & 01 & 04 \\
$30 "$ & - & 04 & 02 & 06 \\
$31 "$ & - & 01 & 01 & 02 \\
$32 "$ & - & 05 & - & 05 \\
$33 "$ & - & 02 & - & 02 \\
$34 "$ & - & 02 & - & 02 \\
$35 "$ & - & 02 & - & 02 \\
$36 "$ & - & 03 & - & 03 \\
$37 "$ & - & - & - & - \\
$38 "$ & - & 01 & - & 01 \\
$39 "$ & - & 01 & - & 01 \\
$40 "$ & - & 01 & - & 01 \\
$41 "$ & - & 01 & - & 01 \\
\hline Total & & 46 & 08 & 54 \\
& & & & \\
\hline
\end{tabular}


Table 3. showing the age distribution of the condensed doctors who obtained post graduate qualifications.

\begin{tabular}{|c|c|c|c|c|c|c|c|c|c|}
\hline \multirow{2}{*}{\multicolumn{3}{|c|}{ Age of 1st P.G. }} & Male & \multicolumn{3}{|c|}{ Female } & \multicolumn{3}{|c|}{ Total } \\
\hline Qua & & & & - & & & & & \\
\hline 30 & Years & - & 04 & & 01 & & $=$ & 05 & \\
\hline 31 & $n n$ & - & 02 & & - & & $=$ & 02 & \\
\hline 32 & $n$ & - & 02 & - & - & & $=$ & 02 & \\
\hline 33 & " " & - & 06 & & - & - & $=$ & 06 & \\
\hline 34 & " " & - & 01 & & - & & $=$ & 01 & \\
\hline 35 & $n$ & - & 05 & & - & $=$ & $=$ & 05 & \\
\hline 36 & " " & - & 01 & & 02 & & $=$ & 03 & \\
\hline 37 & $"$ & - & 05 & & - & & $=$ & 05 & \\
\hline 38 & " " & - & 03 & & 01 & & $=$ & 04 & \\
\hline 39 & $" n$ & - & - & & 02 & - & $=$ & 02 & \\
\hline 40 & $n "$ & - & $\mathrm{O} 2$ & & - & & $=$ & 02 & \\
\hline 41 & $m$ & - & 01 & & - & & $=$ & 01 & \\
\hline 42 & $n "$ & - & 04 & & 01 & & $=$ & 05 & $\ldots$ \\
\hline 43 & " " & - & 02 & & - & & $=$ & 02 & $\cdots$ \\
\hline 44 & $"$ & - & 03 & & - & & $=$ & 03 & \\
\hline 45 & $n "$ & - & 02 & & 01 & & $=$ & 03 & \\
\hline 46 & " " & - & 01 & & 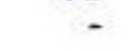 & & $=$ & 01 & \\
\hline 47 & $" n$ & - & 01 & & . & & $=$ & 01 & \\
\hline $48-5$ & $52 "$ & - & - & & 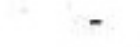 & & $=$ & . - & \\
\hline & " " & - & 01 & & $=$ & & $=$ & 01 & \\
\hline Tot: & & & 46 & & 08 & & $=$ & 54 & \\
\hline
\end{tabular}

Table 4. Shows the time gaps between different Qualifying Examinations

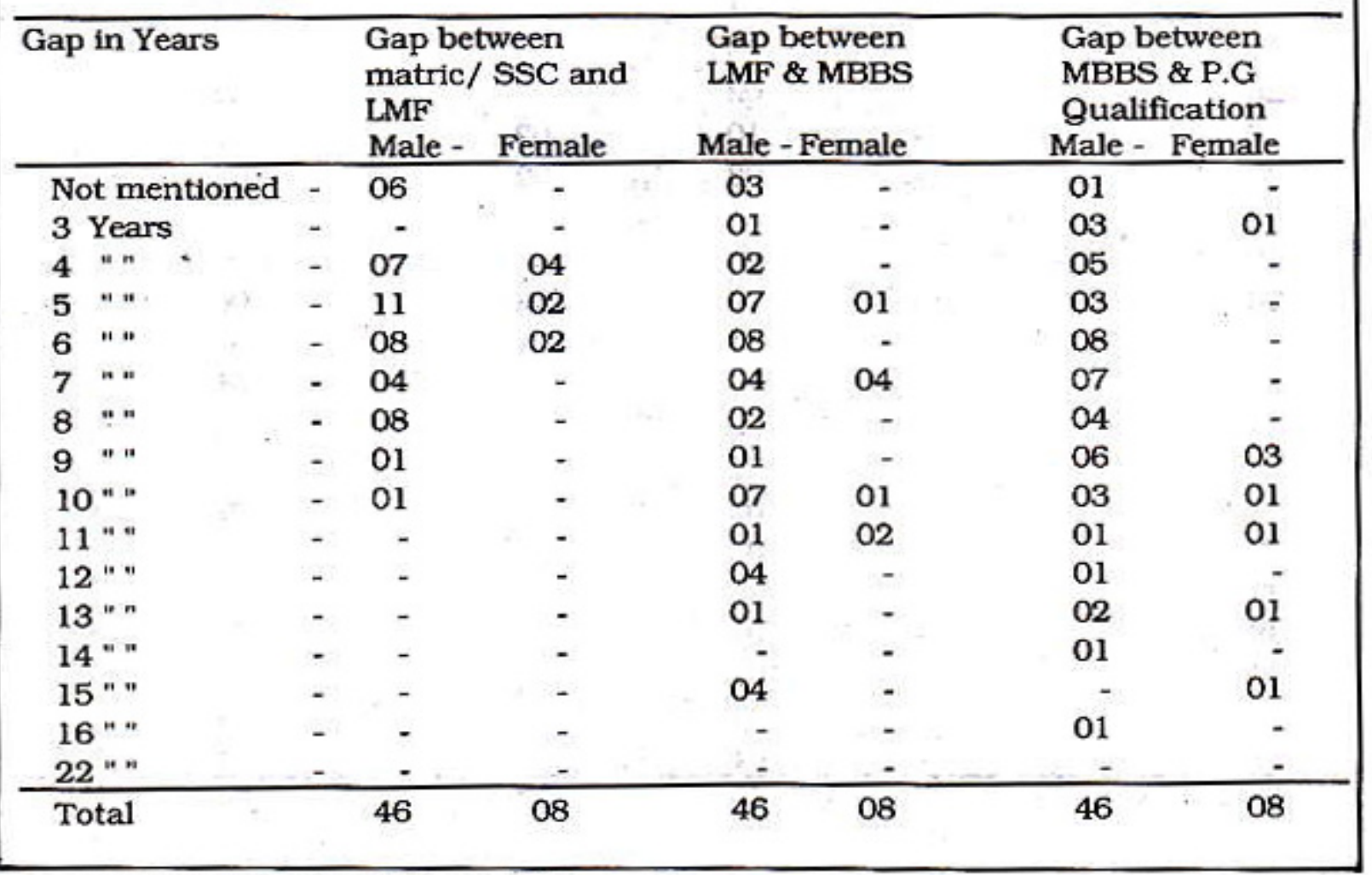


Table 5. showing the age of marriage of the 54 condensed sperialists

\begin{tabular}{|c|c|c|c|c|c|}
\hline Age of Marsiage & & & Male & Female & Total \\
\hline Nat mentioned & - & 02 & - & $=$ & 02 \\
\hline 17 Years & - & 01 & - & $=$ & 01 \\
\hline 18 " n & - & $\infty 2$ & $0 !$ & $=$ & 03 \\
\hline $19 "$ & - & 01 & 01 & $=$ & 02 \\
\hline $20 *$ & . & 01 & - & $=$ & 01 \\
\hline $21 "$ & - & 01 & 01 & $=$ & $\mathrm{O} 2$ \\
\hline $22^{\prime \prime}$ & . & 02 & 02 & $=$ & 04 \\
\hline $23 \cdots$ & - & - & D] & $=$ & 01 \\
\hline $24^{n "}$ & - & 03 & - & $=$ & 03 \\
\hline $25 " "$ & - & 05 & - & $=$ & 05 \\
\hline $26^{\prime \prime}$ & . & 02 & - & $=$ & 02 \\
\hline $27 \cdots$ & - & 04 & - & $=$ & 04 \\
\hline $28 \cdots$ & - & 05 & - & $=$ & 05 \\
\hline $29 \cdots$ & - & 02 & 01 & $=$ & 05 \\
\hline $30 \cdots$ & - & 02 & 01 & $=$ & 03 \\
\hline $31 "$ & - & 05 & - & $=$ & 03 \\
\hline $32 \times n$ & - & 02 & - & $=$ & 02 \\
\hline $33^{n n}$ & - & 04 & - & $=$ & 04 \\
\hline $34 " "$ & - & 01 & - & $=$ & 01 \\
\hline $35 "+$ & - & 으. & - & $=$ & 01 \\
\hline Total & & 46 & 08 & $=$ & 54 \\
\hline
\end{tabular}

Table 6 . showing the member of chtldren of 54 condensed Medical Specialists

\begin{tabular}{|c|c|c|c|c|c|c|c|c|}
\hline \multirow{2}{*}{\multicolumn{2}{|c|}{$\begin{array}{l}\text { Number of } \\
\text { children per } \\
\text { family }\end{array}$}} & \multirow{2}{*}{$\begin{array}{l}\text { No of } \\
\text { Famlly }\end{array}$} & \multirow{2}{*}{$\begin{array}{c}\text { Sex } \\
\text { not } \\
\text { mentioned } \\
\end{array}$} & \multirow{2}{*}{$\begin{array}{c}\text { Sex } \\
\text { mentioned }\end{array}$} & \multicolumn{2}{|c|}{ Sex of children } & \multirow[t]{2}{*}{ Total } & \multirow{2}{*}{$\begin{array}{r}\text { Grand } \\
\text { Total }\end{array}$} \\
\hline & & & & & Mate & Female & & \\
\hline \multirow{2}{*}{\multicolumn{2}{|c|}{$\begin{array}{l}\text { No child } \\
\text { One child }\end{array}$}} & 03 & - & 03 & - & - & $\infty$ & $\infty$ \\
\hline & & 04 & 02 & 02 & 02 & - & 02 & 04 \\
\hline \multicolumn{2}{|c|}{ Two children } & 06 & - & 06 & 06 & 06 & 12 & 12 \\
\hline \multirow{2}{*}{\multicolumn{2}{|c|}{ Three " }} & 16 & OB & - & - & - & 09 & \\
\hline & & & & 13 & 18 & 21 & 39 & 48 \\
\hline \multirow[t]{2}{*}{ Four } & $"$ & 14 & 10 & - & - & - & 40 & \\
\hline & & & & 04 & 10 & 06 & 16 & 56 \\
\hline \multirow[t]{2}{*}{ Fyve } & " & 07 & 05 & $\cdot$ & - & - & 25 & 35 \\
\hline & & & 02 & 08 & 02 & 10 & & \\
\hline Six & $"$ & 02 & 01 & - & - & - & 06 & 12 \\
\hline Seven & $"$ & Ol & 01 & - & - & - & 07 & 07 \\
\hline Eight & $\because$ & 01 & 01 & - & - & - & 08 & 08 \\
\hline \multicolumn{2}{|l|}{ Total } & 54 & 23 & 31 & 48 & 37 & 182 & 182 \\
\hline
\end{tabular}




\section{Conclusion :}

The condensed course inspired a large number of LMF doctors to obtain MBBS degree and 54 of them obtained post graduate qualifications. Since MBBS doctors are reluctant to go to villages. special attractive measures in the form of special allowance, free residence and prevision of standard diognostic laboratory at least in the each Thana town, will inspire the doctors to work in villages, where medical care is very poor.

\section{Reference :}

Muazzam, M.G (1986) : Medical Specialist Directory, Bangladesh, Ist edn.

Ibn Sina Trust, Dhaka.

"Say : O Allah! Lord of Power,

You give power to whom You please.

And You strip off power from

whom You please;

And You exalt whom You please.

And You abase whom You please.

In Your hand is the good.

Verily, Over all the things

You have powers."

- (Al-Gur'an, $3: 26$ ) 